Pesq. Vet. Bras. 37(8):877-882, agosto 2017

\title{
Vascularização uterina de éguas prenhes de jumento e de garanhão monitoradas por ultrassonografia Doppler ${ }^{1}$
}

\author{
Sabina G.C. Lemos²*, Diego G. Campos ${ }^{3}$, Camila S.C. Ferreira ${ }^{4}$, Mário F.A. Balaro ${ }^{5}$, \\ Luís E.R. Cunha ${ }^{6}$, Aline E. Pinna ${ }^{7}$ e Felipe Z. Brandão ${ }^{8}$
}

\begin{abstract}
Lemos S.G.C., Campos D.G., Ferreira C.S.C., Balaro M.F.A., Cunha L.E.R., Pinna A.E. \& Brandão F.Z. 2017. [Uterine vasculature of pregnant mares of donkey and stallion through the Doppler ultrasonography spectral mode.] Vascularização uterina de éguas prenhes de jumento e de garanhão monitoradas por ultrassonografia Doppler. Pesquisa Veterinária Brasileira 37(8):877-882. Faculdade de Veterinária, Universidade Federal Fluminense, Rua Vital Brazil Filho 64, Vital Brazil, Niterói, RJ 24230-340, Brazil. E-mail: binagaiacl@gmail.com

This study aimed to evaluate and describe the ultrasonographic findings of uterus of pregnant mares of different ages, mated by either ass or stallion, using the spectral Doppler ultrasonography, aiming to characterize the resistance (RI) and pulsatility (PI) Doppler indices up to 154 days of pregnancy. A total of 20 mares in reproductive age of non-defined breed were used. The evaluations were performed in uterine arteries. There was no interaction between RI and PI values obtained in uterine horns (that initiated or not the pregnancy) with time of pregnancy ( $\mathrm{P}>0.05)$, thus, data were discussed separately. There were no differences between RI and PI evaluations when compared to the uterine horns that initiated or not the pregnancy $(\mathrm{P}>0.05)$. A time effect was observed $(\mathrm{P}<0.05)$ on $\mathrm{PI}$, when D70 presented lower averages than D0, D7, D14 and D21. There was no time effect $(\mathrm{P}>0.05)$ on RI. Pregnant mares mated by asses presented higher uterine vascularization in non-pregnant horn than pregnant mares mated by stallions. With the exception of RI in non-pregnant horn, there was a female age effect on the side of pregnancy $(\mathrm{P}<0.05)$, where aged mares in both - pregnant and non-pregnant horns - showed higher PI and RI, so less vascularized. It is possible to conclude that the uterine vascularization did not present any difference between uterine horns. There is an increase in the uterine vascularization on the 70th day of pregnancy. Pregnant mares mated by assess presented higher uterine vascularization when compared to those mated by stallions. Mares aging over 15 years old presented lower uterine vascularization, when compared to those younger.
\end{abstract}

INDEX TERMS: Mare, ultrasound, Doppler, spectral waves, vascularization, uterus.

RESUMO.- Este estudo teve como objetivo analisar e descrever os achados ultrassonográficos do útero de éguas gestantes de diferentes idades, de jumento e garanhão, utilizando a técnica de ultrassonografia Doppler Espec-

\footnotetext{
${ }^{1}$ Recebido em 2 de fevereiro de 2016.

Aceito para publicação em 6 de dezembro de 2016.

${ }^{2}$ Programa de Pós-Graduação em Medicina Veterinária, Faculdade de Veterinária, Universidade Federal Fluminense (UFF), Rua Vital Brazil Filho 64, Vital Brazil, Niterói, RJ 24230-340, Brasil. *Autor para correspondência: binagaiacl@gmail.com

${ }^{3}$ Programa de Pós-Graduação em Ciências Veterinária, Universidade Federal Rural do Rio de Janeiro (UFRRJ), Rodovia BR-465, Km 7, Seropédica, RJ 23851-970, Brasil. E-mail: diegoguedescampos@hotmail.com

${ }^{4}$ Faculdade de Veterinária, UFF, Rua Vital Brazil Filho 64, Vital Brazil, Niterói, RJ 24230-340, Brasil. E-mail: vetcamilaferreira@gmail.com
}

tral, visando caracterizar os índices de resistência (RI) e pulsatilidade (PI) até 154 dias de gestação. Foram utilizadas 20 éguas em idade reprodutiva, sem raça definida. As avaliações foram realizadas nas artérias uterinas. Não foi

\footnotetext{
${ }^{5}$ Departamento de Patologia e Clínica Veterinária, Faculdade de Veterinária, UFF, Rua Vital Brazil Filho 64, Vital Brazil, Niterói, RJ 24230-340, Brasil. E-mail: mariobalaro@hotmail.com

${ }^{6}$ Instituto Vital Brazil S/A, Rua Maestro José Botelho 64, Vital Brazil, Niterói, RJ 24230410, Brasil. E-mail: luis.eduardo@vitalbrazil.rj.gov.br

${ }^{7}$ Departamento de Patologia e Clínica Veterinária, Faculdade de Veterinária, UFF, Rua Vital Brazil Filho 64, Vital Brazil, Niterói, RJ 24230-340, Brasil. E-mail: aepinna@id.uff.br

${ }^{8}$ Programa de Pós-Graduação em Medicina Veterinária, UFF, Rua Vital Brazil Filho 64, Vital Brazil, Niterói, RJ 24230-340, Brasil. E-mail: fzbr@vm.uff.br
} 
constatada interação entre os valores de RI e PI obtidos nos cornos uterinos (que iniciaram ou não a gestação) com o tempo da gestação $(\mathrm{P}>0,05)$, desta forma os dados foram discutidos separadamente. Não foram encontradas diferenças entre as avaliações de RI e PI quando comparado os cornos que iniciaram ou não a gestação $(P>0,05)$. Verificou-se efeito de tempo $(\mathrm{P}<0,05)$ sobre o PI, onde no D70 foi constatada a menor média quando comparado aos tempos D0, D7, D14 e D21. Não f oi encontrado efeito de tempo sobre o RI $(P>0,05)$. As éguas gestantes de jumento apresentaram maior vascularização uterina no corno não gestante, que as éguas gestantes do garanhão. Com exceção do RI no lado não gravídico, foi verificado efeito de idade da fêmea sobre o lado da gestação $(\mathrm{P}<0,05)$, onde as éguas idosas, tanto no corno que iniciou a gestação e o contralateral, tiveram PI e RI mais altos, ou seja, menos vascularizados. Concluiu-se que a vascularização uterina não apresentou diferença entre os cornos uterinos. Há um aumento da vascularização uterina no $70^{\circ}$ dia de gestação. Éguas gestantes de jumentos apresentaram maior vascularização uterina, quando comparada às éguas gestantes de garanhões. Éguas com idade acima de 15 anos apresentaram menor vascularização uterina, quando comparadas com idade inferior a 15 anos.

TERMOS DE INDEXAÇÃO: Éguas, ultrassom, Doppler, ondas espectrais, vascularização, útero.

\section{INTRODUÇÃO}

Os asininos (Equus asinus) estão conquistando o mercado brasileiro. Segundo Blanchard \& Taylor (2005), os asininos e muares (produtos híbridos do cruzamento entre asininos e equinos) estão desfrutando de crescente popularidade como animais de estimação e animais de trabalho. Canisso et al. (2009) confirmam essa informação ao afirmarem que, no Brasil, tem havido grande interesse em jumentos com especial foco na doação de sêmen para a produção de muares, embora existam no país plantéis asininos de elevado padrão genético, onde o reprodutor é utilizado para a melhoria zootécnica da própria espécie.

Antigamente os muares eram considerados apenas como animais de "carga", mas, com o passar dos anos, tem havido crescimento no número de mulas e burros de alto valor zootécnico. Conforme explicam Torres \& Jardim (1992), estes animais são provenientes do cruzamento de éguas e jumentos de alto valor genético, o que os torna mais valorizados, considerados assim pelos criadores como animais completos, apresentando qualidade, funcionalidade e beleza zootécnica.

Por este motivo, o estudo da gestação destes híbridos ganha cada vez mais importância, surgindo questões sobre haver ou não diferenças entre a gestação de potros e muares no útero da égua. Em relação à gestação na égua, sabe-se que, durante seu período inicial, conforme Silva et al. (2005), uma íntima interação entre o concepto e o endométrio ao longo de toda a extensão do útero é necessária para o adequado reconhecimento embrionário e inibição da regressão prematura do corpo lúteo. Durante este período inicial, a vascularização uterina sofre alterações devido à mobilidade embrionária. Há migração da vesícula embrio- nária pelo útero e já foi demonstrado que a vascularização acompanha essa migração até o momento da fixação do concepto. Posteriormente a esse reconhecimento embrionário, a irrigação sanguínea, pela artéria uterina, se difunde por todo útero conforme o crescimento fetal se estende. Através desta, os anexos fetais e placentários, também recebem aporte sanguíneo, e durante a gestação ocorrem inúmeros eventos como a formação dos anexos fetais e placentação. Desta forma, Ferreira et al. (2008) afirmam que uma melhor compreensão da interação embrio-maternal e das possíveis alterações endometriais sobre o desenvolvimento do embrião podem ser obtidos através da Ultrassonografia (US) Doppler.

Paralelamente a questão da gestação de híbridos, outra variável que pode influir na manutenção desta gestação é a idade da égua. Conforme Carnevale \& Ginther $(1992,1995)$, são consideradas éguas idosas aquelas com idade igual ou superior a 15 anos, havendo uma diminuição da fertilidade de éguas a partir desta idade. Essa diminuição tem sido atribuída a diversos fatores, como o decréscimo da atividade ovariana e da contratilidade uterina, o que dificulta a eliminação de bactérias e corpos estranhos, prolongado período interovulatório, menor qualidade do oócito e menor atividade folicular durante o período de transição.

Assim, torna-se de fundamental importância maiores estudos sobre a gestação de éguas com jumentos, fazendo um comparativo com a gestação de garanhões, para identificar possíveis diferenças entre elas, o que pode ser feito através de métodos diagnósticos como a US Doppler, para detectar a ocorrência de alterações na vascularização no ambiente uterino da gestante, permitindo o diagnóstico precoce de mudanças, com resultados imediatos, sem a utilização de procedimentos invasivos e podendo ser usado como ferramenta de rotina.

Na ultrassonografia Doppler modo espectral, a avaliação hemodinâmica do útero pode ser feita a partir dos grandes vasos, como as artérias uterinas (Bollwein et al. 1998). Silva et al. (2005) recomendam o uso dos índices de pulsatilidade (PI) e de resistência (RI), já que ambos são bons indicadores da perfusão vascular dos órgãos reprodutivos e não sofrem influencia do ângulo Doppler. Desse modo, o aumento dos valores de PI e de RI indica um aumento na resistência e, consequentemente, uma diminuição no fluxo sanguíneo no interior do vaso.

Dessa forma, este estudo teve como objetivo avaliar a vascularização uterina em éguas jovens e idosas gestantes de jumento e garanhão por meio da US Doppler modo Espectral, do D0 até o D154 de gestação, visando demonstrar a existência de diferenças entre as gestações durante esse período.

\section{MATERIAL E MÉTODOS}

O estudo foi aprovado pelo Comitê de Ética no Uso de Animais da Universidade Federal Fluminense (projeto número 309/14).

\section{Localização e período experimental}

0 experimento foi realizado nas instalações da Fazenda Vital Brazil, pertencente ao Instituto Vital Brazil, situada no Km 23, da estrada RJ 122, no município de Cachoeiras de Macacu, localizado no Estado do Rio de Janeiro. A propriedade encontra-se na latitu- 
de $22^{\circ} 27^{\prime} 45^{\prime \prime}$ sul e longitude $42^{\circ} 39^{\prime} 11^{\prime \prime}$ oeste, a uma altitude média de $57 \mathrm{~m}$. 0 experimento foi conduzido entre os meses de outubro a julho de 2014, correspondente à estação de monta 2013/2014.

\section{Animais e condições experimentais}

Éguas. Para o experimento, foram selecionadas 20 éguas em idade reprodutiva, sem raça definida, sendo descartadas éguas arredias, agitadas ou com potro-ao-pé devido à dificuldade de realização da técnica de US Doppler. As 20 éguas mantiveram a gestação até o término do estudo (154 dias de gestação).

Antes do início do estudo, as éguas foram submetidas ao exame ginecológico avaliando-se a conformação externa e interna do trato genital. Foi realizado todo o processo de coleta de sêmen, controle folicular das éguas e inseminação artificial daquelas que se encontravam em estro e estado hígido. Para o manejo reprodutivo das éguas seguiu-se um organograma de controle reprodutivo semelhante ao proposto por Palhares (1987), modificado de forma a atender os objetivos do trabalho.

Quanto ao manejo nutricional dos animais durante o experimento, este consistiu no fornecimento da alimentação por meio do método "espinha de peixe", o qual permitia que os animais permanecessem em troncos individuais e assim com acesso a cochos individuais. Cada animal recebia uma dieta fracionada em duas refeições diárias, com os seguintes componentes: $3 \mathrm{~kg}$ de ração comercial, $8 \mathrm{~kg}$ de capim elefante (Pennisetum purpureum) picado, $1 \mathrm{~kg}$ de farelo de trigo (Triticum vulgare vill) ou $400 \mathrm{~g}$ de malte (Hordeum vulgare) e $150 \mathrm{~g}$ de sal mineral comercial e água ad libitum. Na parte da tarde, após o término das atividades, os animais eram soltos em piquetes, se alimentando de Brachiaria humidicola ou Tifton e somente retornavam no dia seguinte para o manejo, permitindo a manutenção do bem-estar animal.

Reprodutores. Utilizou-se um reprodutor asinino e um equino como doadores de sêmen. 0 asinino da raça Pêga com 18 anos de idade e o garanhão da raça Mangalarga Marchador com 10 anos. Os animais foram submetidos ao exame andrológico antes do início do experimento, através de inspeção e palpação da genitália externa, além da avaliação do comportamento sexual, não apresentando disfunção orgânica ou reprodutiva. Após cada colheita de sêmen eram avaliadas as características do ejaculado como concentração, motilidade e vigor. Somente foram utilizados ejaculados que atendiam as recomendações do CBRA (2013).

0 sêmen era coletado e as inseminações foram realizadas quando havia a presença em um dos ovários de um folículo com 3,0 a 3,5 cm de diâmetro.

Após a coleta do sêmen, este era levado para o laboratório, localizado dentro da própria fazenda para sua avaliação. A diluição era realizada utilizando-se o diluidor comercial BotuSemen (Botupharma Biotecnologia Animal LTDA, Botucatu, São Paulo, Brasil). A inseminação artificial era realizada no corpo do útero, com a égua contida no tronco. Realizava-se a limpeza da região perineal da égua para efetuar o procedimento da inseminação.

\section{Desenho do estudo}

Após a confirmação da gestação, as fêmeas foram agrupadas da seguinte forma: Tipo de acasalamento: foram agrupadas de acordo com o cruzamento utilizado, sendo gestantes de garanhão $(n=9)$ e gestantes de jumento $(n=11)$. Faixa etária: éguas consideradas jovens com menos de 15 anos (idade média 9,46 $\pm 2,53$ anos - $\mathrm{n}=10$ ) e éguas idosas, com idade acima de 15 anos (idade média $17,81 \pm 1,16$ anos $-n=10$ ).

\section{Ultrassonografia Doppler}

Foram realizados exames ultrassonográficos, utilizando-se um aparelho de ultrassom da marca SonoScape modelo S6 (Shenzhen, China), equipado com um transdutor linear transretal de 5,0 a 10,0 Mhz. Os exames foram realizados inicialmente no modo B e em seguida pela função power-flow e modo espectral.

Para a realização do diagnóstico de gestação no 14ํ dia após a ovulação, usava-se apenas o Modo B. Através da presença da vesícula embrionária e ainda do corpo lúteo no ovário que ocorreu a ovulação, confirmava-se a gestação, e assim a égua era avaliada semanalmente até o $154^{\circ}$ dia da gestação.

Foi utilizado o Doppler colorido para avaliar a presença, a direção e a qualidade do fluxo sanguíneo do útero. A programação do equipamento foi mantida constante ao longo do estudo. A frequência de repetição de pulsos (PRF) utilizada para o modo Espectral foi de $8.0 \mathrm{~Hz}$ e de $1.0 \mathrm{~Hz}$ para o modo Doppler.

Foram registrados os dados do Doppler Modo espectral, utilizando os índices de RI e de PI. Estas avaliações foram realizadas nas artérias uterinas através do transdutor linear transretal, com o transdutor direcionado para o dorso do animal, onde se encontram os vasos (Bollwein et al. 1998).

Ao localizar a artéria ilíaca externa movia-se o transdutor de um lado a outro, longitudinalmente à aorta a procura das artérias uterinas direita e esquerda (corno gestante e corno não gestante), e a partir destas foram obtidas ondas espectrais através dos ciclos cardíacos gerados. Posteriormente foram selecionadas três ondas de aspecto semelhante. Foram retirados manualmente do aparelho os valores dos índices Doppler para o cálculo das médias, considerando esta ao valor mais próximo da segunda onda avaliada.

Cada égua possuía uma ficha, em que foram preenchidas informações necessárias à interpretação dos dados obtidos, como o histórico reprodutivo, a idade dos animais, os achados físicos e os de ultrassonografia.

Análise estatística: Os resultados obtidos foram analisados por meio de programa estatístico próprio (Sistema para Análises Estatísticas - SAEG ${ }^{\circledR}$ 9.0, UFV, Viçosa, Brasil). Adotou-se uma análise descritiva do comportamento temporal de PI e RI. Inicialmente, o teste de Lilliefors foi adotado para verificar a normalidade de todas as variáveis quantitativas estudadas. Na sequência, aplicou-se a análise de variância - ANOVA para modelos lineares e a comparação de médias pelo Teste de Tukey $(\mathrm{P}<0,05)$.

\section{RESULTADOS E DISCUSSÃO Efeito do local de ocorrência da gestação e tempo da gestação}

Não houve interação entre o corno uterino gestante ou não e o tempo da gestação. Sendo assim, os dados serão discutidos separadamente, ou seja, inicialmente em relação ao corno uterino e posteriormente em relação ao tempo de avaliação (Fig.1). Os valores médios de PI e RI das artérias uterinas de acordo com o corno uterino gestante e contralateral à gestação das éguas, independente do cruzamento e nos tempos de avaliação, respectivamente, foram de 1,84 $\pm 0,02$ vs $1,80 \pm 0,02$ e $0,80 \pm 0,03$ vs $0,81 \pm 0,04(\mathrm{P}>0,05)$.

Silva et al. (2005) realizando avaliação subjetiva dos vasos mesometriais de éguas gestante com diferentes idades, relataram que o corno contralateral à gestação apresentava vascularização intensa, porém menor em relação ao corno gestante. No presente estudo não houve diferença entre os dois cornos, utilizando o modo espectral das artérias uterinas, sugerindo assim a avaliação modo Doppler colorida, anteriormente citada, mais fidedigna para este estudo específico. Reynolds et al. (1984) utilizando a técnica de implantação de transdutores eletromagnéticos nas artérias uterinas de ovelhas, demonstraram que o fluxo sanguíneo aumentou nas artérias ipsilaterais ao concepto, mas não 


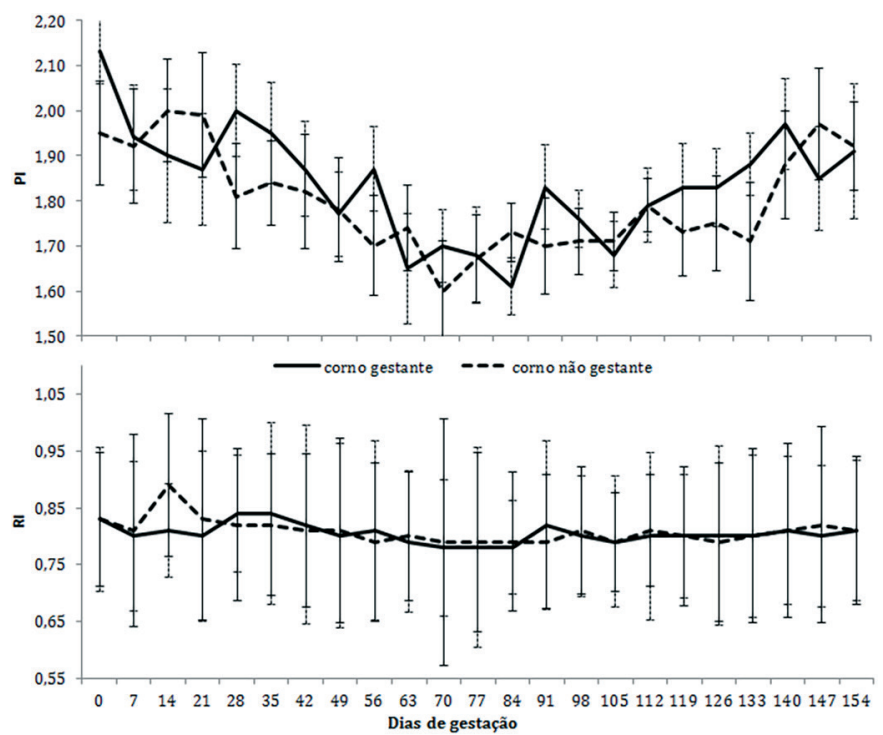

Fig.1. Índice de pulsatilidade (PI) e resistência (RI) das artérias uterinas em corno gestante e não gestante de éguas sem raça definida até 154 dias de gestação (média \pm erro padrão)

na contralateral entre os dias 13 e 15 de gestação. Ford et al. (1979) igualmente obtiveram as mesmas respostas nos dias 15 e 17 de gestação em vacas. Ford \& Christenson (1979) verificaram que em suínos, quando os embriões foram confinados experimentalmente em um corno, o fluxo de sangue aumentou somente neste lado. Os mesmos autores observaram quando embriões suínos estavam presentes em ambos os cornos uterinos, que o fluxo de sangue aumenta transitoriamente em ambas as artérias uterinas nos dias 12 e 13 após a inseminação, sendo utilizadas sondas de fluxo sanguíneo eletromagnéticas, colocadas ao redor de ambas as artérias uterinas. Ford et al. (1979) conduzindo um experimento com sondas eletromagnéticas avaliaram o fluxo de sangue para o útero gravídico em novilhas, onde observaram aumento do fluxo na artéria uterina ipsilateral ao embrião propriamente dito, nos dias 14-18 e depois do $25^{\circ}$ dia. Os resultados encontrados no presente estudo podem ser justificados pela metodologia utilizada (modo espectral) e pelo fato da diferença na fisiologia do reconhecimento materno da gestação em éguas, pois a vesícula embrionária migra por toda extensão uterina até $16^{\circ}-17$ 음 dia de gestação e a vascularização acompanha tal migração, não levando assim, diferença entre os cornos avaliados, justificando desta forma os resultados encontrados.

Alterações de fluxo sanguíneo na artéria uterina foram estudadas por ultrassonografia Doppler em vacas ao longo da gestação (Bollwein et al. 2002). Estes observaram que o índice de resistência (RI) foi menor e o volume de fluxo sanguíneo foi mais elevado na artéria ipsilateral ao concepto, fato não observado no presente estudo.

Quando da avaliação ao longo da gestação (Fig.2), no presente estudo, verificou-se efeito de tempo $(\mathrm{P}<0,05)$ somente sobre o PI, não foi encontrado efeito de tempo sobre o RI $(\mathrm{P}>0,05)$. No D70 foi constatada a menor média quando comparado aos tempos D0, D7, D14 e D21. Panarace et al. (2006) estudaram uma vez por semana, como no presente estudo, 13 vacas gestantes entre os dias $30^{\circ}-270^{\circ}$

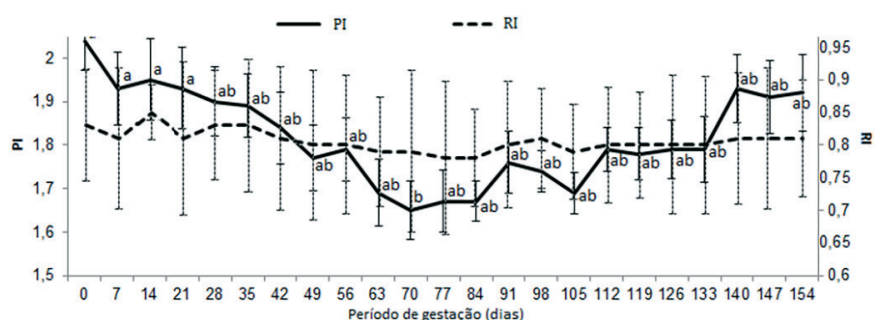

Fig.2. Índice de pulsatilidade (PI) e resistência (RI) das artérias uterinas independente do corno uterino de éguas sem raça definida até 154 dias de gestação (média \pm erro padrão)
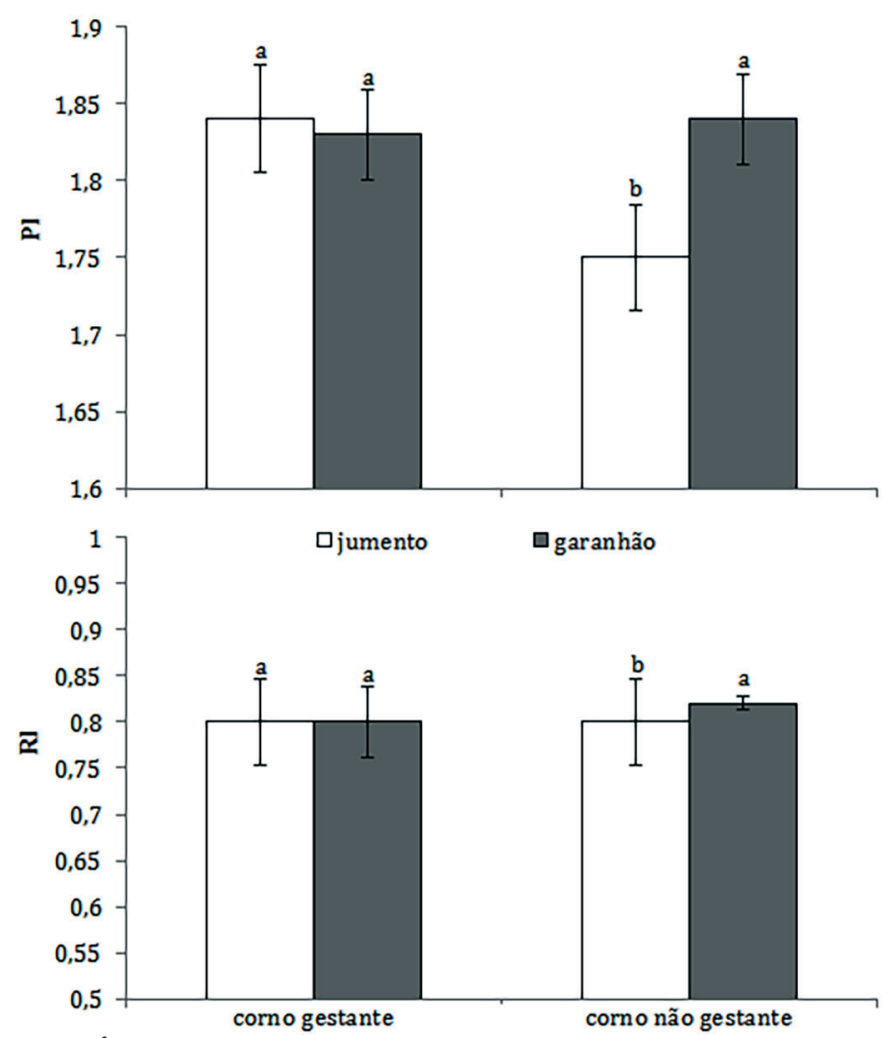

Fig.3. Índice de pulsatilidade (PI) e resistência (RI) das artérias uterinas, em corno gestante e não gestante de éguas sem raça definida, cobertas por jumento ou garanhão, até 154ํ dia de gestação (média \pm erro padrão)

de gestação. Os autores observaram que os valores de RI nas artérias uterinas diminuíram continuamente durante a gestação, o que não ocorreu no presente estudo, onde não foi encontrado efeito do tempo da gestação sobre o RI $(\mathrm{P}>0,05)$.

Panarace et al. (2006) observaram um grande aumento no volume de sangue após 16 semanas de gestação no lado do corno gestante e um ligeiro aumento do volume de sangue foi observado após 20 semanas no corno contralateral a gestação, como observado no presente estudo, onde foi determinado um aumento na vascularização com o pico máximo na 10a semana (D70). Clegg et al. (1954) demonstraram que os cálices endometriais nas éguas gestantes tem sua produção máxima de eCG aproximadamente entre os dias 60-75 de gestação. Como no presente estudo a avaliação foi feita semanalmente (63-70-77 dias de gestação) igualmente poderia sugerir um aumento da perfusão vas- 
cular uterina, uma vez que este hormônio é uma substância vasoativa e estaria atuando no trato reprodutivo do animal.

\section{Efeito do reprodutor utilizado}

Os resultados obtidos nas avaliações de PI e RI das artérias uterinas, de acordo com o lado da ocorrência da gestação e do acasalamento utilizado (jumento e garanhão), encontram-se na Figura 3. Não foi observado efeito do tempo da gestação $(\mathrm{P}>0,05)$ sobre as respostas estudadas. Foi encontrado somente efeito do reprodutor utilizado no acasalamento sobre os valores de RI e PI do corno uterino em que a vesícula não iniciou o seu desenvolvimento, onde éguas gestantes de jumento apresentaram menores valores de PI e RI $(\mathrm{P}<0,05)$. Whitwell \& Leffcott $(1975)$ observaram que os vasos sanguíneos fetais da égua gestante se espalham sobre toda superfície do corioalantóide e que os vasos do cordão umbilical invadem o corno não gravídico. No presente estudo, este evento ocorreu mais intensamente nas gestações oriundas do acasalamento com o jumento. Carluccio et al. (2008) observaram que o corioalantóide de jumentas tem um padrão de ramificação dos microcotiledones mais complexa, o que pode sugerir um fluxo sanguíneo maior para esta região, explicando assim os resultados encontrados.

\section{Efeito da idade}

Quanto ao efeito da idade da égua em relação às avaliações ultrassonográficas, não foi constatada a interação entre o corno uterino de ocorrência da gestação em relação
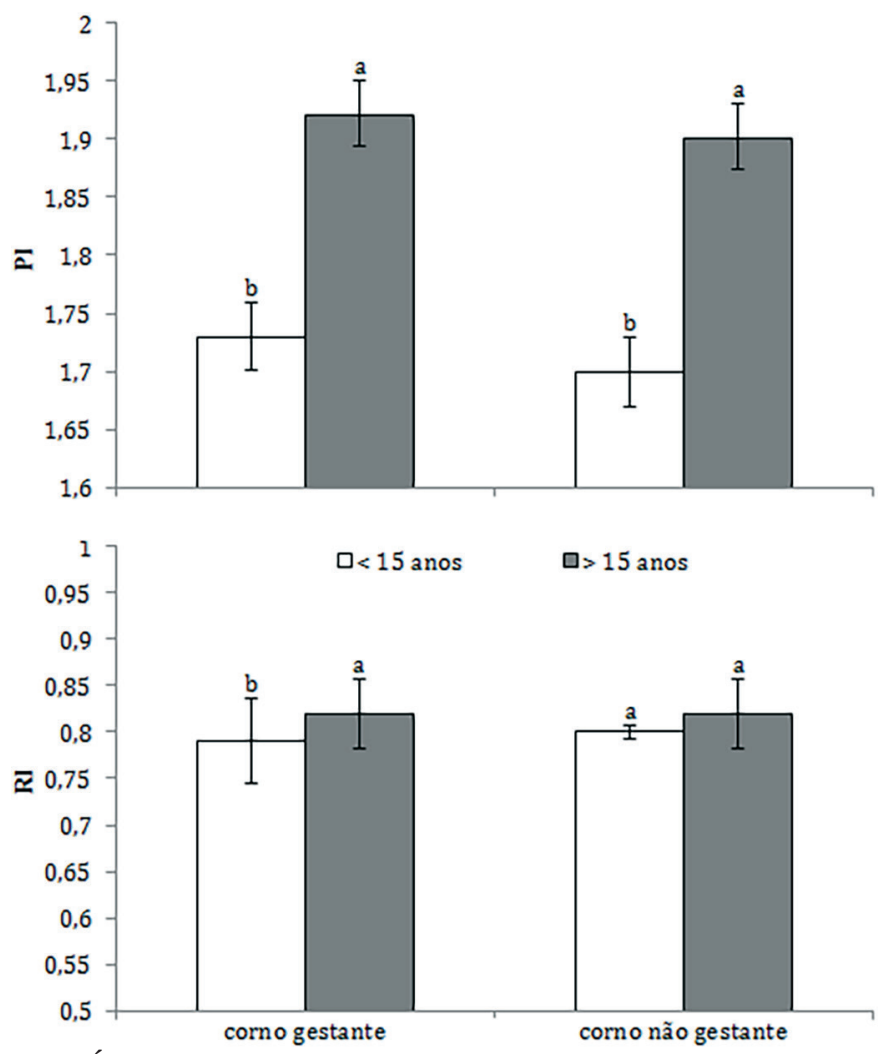

Fig.4. Índice de pulsatilidade (PI) e Resistência (RI) das artérias uterinas em corno gestante e não gestante de éguas sem raça definida com idade abaixo e acima de 15 anos, até 154ํ dia de gestação (média \pm erro padrão) ao tempo da gestação $(\mathrm{P}>0,05)$. Com exceção do RI no lado não gravídico, foi verificado efeito de idade da fêmea sobre os valores obtidos nos cornos uterinos avaliados $(\mathrm{P}<0,05)$, onde foi observado maior índice resistência e pulsatilidade nas éguas acima de 15 anos. Observam-se, na Figura 4, os dados relativos ao PI e RI das artérias uterinas, em corno gestante e não gestante das éguas jovens ( $<15$ anos) e idosas ( $>15$ anos) independentemente do tempo de gestação.

0 presente estudo encontrou alterações hemodinâmicas ao comparar PI e RI de éguas idosas com éguas jovens, apontando para um menor fluxo sanguíneo nas primeiras, da mesma forma que Bollwein et al. (1998) e Ferreira et al. (2008) em seus estudos, onde verificaram o aumento na resistência vascular em éguas com idade avançada. Segundo Bollwein et al. (1998), estas alterações podem ser atribuídas a fibrose, e da mesma forma já foram encontradas em mulheres, conforme explicam Cacciatore et al. (1996), estando relacionadas com problemas de infertilidade. Allen (1992) demonstrou que as patologias mais significativas que alteram a morfologia e função uterina em éguas idosas são o bloqueio dos canais linfáticos, deposição de camadas de tecido conjuntivo fibroso em torno das glândulas endometriais e inatividade do útero pela perda do tônus e da contratilidade do miométrio. As alterações degenerativas presentes comumente no útero de éguas idosas justificam a queda da perfusão vascular uterina encontrada no presente estudo.

\section{CONCLUSÕES}

Os resultados obtidos no presente estudo indicam que a vascularização uterina em éguas não apresentou diferença entres os cornos gestantes e não gestantes.

Éguas gestantes de jumentos apresentaram maior vascularização uterina no corno uterino contralateral ao que iniciou a gestação.

Houve um aumento da vascularização uterina no $70^{\circ}$ dia de gestação.

Éguas idosas apresentaram menor fluxo sanguíneo na artéria uterina, quando comparadas às éguas jovens.

Agradecimentos.- Os autores agradecem aos apoios recebidos no Edital InfraLabPesq/Proppi-UFF e da Faperj. FZB e SGCL são bolsistas do CNPq.

\section{REFERÊNCIAS}

Allen W.R. 1992. The diagnosis and handling of early gestacional abnormalities in the mares. Anim. Reprod. Sci. 28(1):31-38.

Blanchard T.L. \& Taylor T.S. 2005. Estrous cycle characteristics of donkeys with emphasis on standard and mammoth donkeys. In: Matthews N.S. \& Taylor T.S. (Eds), Veterinary Care of Donkeys. International Veterinary Information Service (www.ivis.org), Ithaca, New York.

Bollwein H., Maierl J., Mayer R. \& Stolla R. 1998. Transrectal color Doppler sonography of the A. uterina in cyclic mares. Theriogenology 49:14831488.

Bollwein H., Baumgartner U. \& Stolla R. 2002. Transrectal Doppler sonography of uterine blood flow in cows during pregnancy. Theriogenology 57:2053-2061.

Cacciatore B.N., Simber B.N., Fusaro P. \& Tiitinen A. 1996. Transvaginal Doppler study of uterine artery blood flow in vitro fertilization-embryo transfer cycles. Fertility and Sterility 66:130-134.

Canisso I.F., Carvalho G.R., Silva E.C., Rodrigues A.L., Ker P.G. \& Guimarães J.D. 2009. Alguns aspectos biométricos do aparelho genital externo de 
jumentos doadores de sêmen da raça Pega. Ciência Rural 39(9):25562562.

Carluccio A., Panzani S., Tosi U., Riccaboni P., Contri A. \& Veroneso M. 2008. Morphological features of the placenta at term in the Martina Franca donkey. Theriogenology 69(8):918-924.

Carnevale E.M. \& Ginther O.J. 1992. Relationships of age to uterine function and reproductive efficiency in mares. Theriogenology 37(5):11011115.

Carnevale E.M. \& Ginther O.J. 1995. Defective oocytes as a cause of subfertility in old mares. Biol. Reprod., Mono Lake, 1:209-214.

CBRA 2013. Manual para Exame Andrológico e Avaliação de Sêmen Animal. 3a ed. Colégio Brasileiro de Reprodução Animal, Belo Horizonte.

Clegg M.T., Boda J.M. \& Cole H.H. 1954. The endometrial cups and allantochorionic pouches in the mare with emphasis on the source of equine gonadotrophin. Endocrinology 54:448-463.

Ferreira J.C., Gastal E.L. \& Ginther O.J. 2008. Uterine blood flow and perfusion in mares with uterine cysts: effect of the size of the cystic area and age. Reproduction 135:541-550.

Ford S.P., Chenault J.R. \& Echternkamp S.E. 1979. Uterine blood flow of cows during the estrous cycle and early pregnancy: effect of the conceptus on the uterine blood supply. J. Reprod. Fertility 56:53-62.
Ford S.P. \& Christenson R.K. 1979. Blood flow to uteri of sows during the estrous cycle and early pregnancy: local effect of the conceptus on the uterine blood supply. Biol. Reprod. 21:617-624.

Palhares M.S. 1989. Avaliação da atividade ovariana e eficiência reprodutiva de potras e éguas da raça Mangalarga Marchador. Dissertação de Mestrado em Medicina Veterinária, Escola de Veterinária, Universidade Federal de Minas Gerais, Belo Horizonte. 89p.

Panarace M., Garnil C., Marfil M., Jauregui G., Lagioia J., Luther E. \& Medina M. 2006. Transrectal Doppler sonography for evaluation of uterine blood flow throughout pregnancy in 13 cows. Theriogenology 66:21132119.

Reynolds L.P., Magness R.R. \& Ford S.P. 1984. Uterine blood flow during early pregnancy in ewes: interaction between the conceptus and the ovary bearing the corpus luteum. J. Anim. Sci. 58:423-429.

Silva L.A., Gastal E.L., Beg M.A. \& Ginther O.J. 2005. Changes in vascular perfusionof the endometrium in association with changes in location of the embryonic vesicle in mares. Biol. Reprod. 72:755-761.

Torres A.P. \& Jardim W.R. 1992. Criação do Cavalo e de Outros Equídeos. 3a ed. Nobel, São Paulo, SP.

Whitwell K.E. \& Jeffcott L.B. 1975. Morphological studies on the fetal membranes of the normal singleton foal at term. Res. Vet. Sci, 19(1):44-45. 